\title{
NEWCOMB ASTROMETRIC SATELLITE
}

\author{
K. J. JOHNSTON AND P. K. SEIDELMANN \\ U.S. Naval Observatory \\ Washington, D.C. 20392-5420 \\ AND \\ R. D. REASENBERG, R. BABCOCK AND J. D. PHILLIPS \\ Smithsonian Astrophysical Observatory \\ Cambridge, Massachusetts
}

\begin{abstract}
.
Newcomb is a design concept for an astrometric optical interferometer satellite with a nominal single measurement accuracy of 100 microarcseconds. In a 30-month mission life, it will make scientifically interesting measurements of $\mathrm{O}$ stars, RR Lyrae and Cepheid distances, probe dark matter in our Galaxy via parallax measurements of $\mathrm{K}$ giants in the disk, establish a reference grid with internal consistency better than 50 microarcseconds, and lay the groundwork for the larger optical interferometers that are expected to produce a profusion of scientific results during the next century.
\end{abstract}

\section{Concept}

The idea is to achieve an initial optical interferometer in space by means of a small satellite with a simple design, which could be launched by a small rocket, such as the Pegasus, at a low cost to the mission. The spacecraft would hold a stack of simple optical interferometers pointing in certain fixed relative directions. The interferometers will provide high precision differential angle measurements with a nominal single measurement accuracy of 100 microarcseconds. The optics would be mounted on a three-axis gimbal with the spacecraft holding inertial orientation. The plan would be for an orbit of about 600 nautical miles in altitude with continuous sun illumination and a lifetime of two and a half years. 


\section{Justification}

The Newcomb Astrometric Satellite would provide a precise inertial reference frame based on something over 1000 stars observed with an accuracy of 0.5 nanoradian, or 0.1 milliarcsecond, at the epoch of observation. The single instrument would provide all sky coverage. These results are compared to the current optical reference frame, which has an accuracy of 250 nanoradians, or 50 milliarcseconds, and worse when systematic errors are considered. The Hipparcos mission will provide 10 nanoradians accuracy, or 2 milliarcseconds, at the epoch of observation with an error increasing at the same value per year from the epoch of observation. Ground-based interferometer observations are expected to be limited to 10 nanoradians or 2 milliarcseconds accuracy.

Alternative future plans for an optical interferometer in space include POINTS and OSI which are not expected to be able to fly for 15 or 20 years. This is another reason for the Newcomb Astrometric Satellite, as it will provide a method for characterizing materials and techniques for optical interferometry in space, and be a testbed for future DoD or NASA interferometry missions such as AIM or TOPS. Also, this satellite mission will provide technology for calibrating inertial orientation systems and a means for future precise pointing for satellite systems.

\section{Scientific Goals}

Newcomb would provide a direct link between high precision optical astrometry and the radio reference frame (Fey, et al., 1992). Observations of a few radio quasars can fix the rotation between the radio reference frame and the optical reference frame as developed by the Newcomb and Hipparcos observations.

Assuming that the design would develop a limiting magnitude around 15 , there are a number of science studies that could be accomplished. Nineteen of the known Cepheids have parallaxes between 200 and $1000 \mathrm{mi}-$ croarcseconds and visual magnitudes of less than 10 . So a $5 \%$ distance measurement would be useful in refining the cosmic distance scale. Absolute magnitudes of $\mathrm{O}$ stars are uncertain because none is close enough to allow a trigonometric parallax measurement from the ground. Since those stars are bright targets with magnitudes of 4 to 6 at 1 - 2 kiloparsecs, 25 500 microarcsecond parallax measurements are needed.

Age determinations of globular clusters depend, in part, on the calibration of the absolute magnitude of RR Lyrae stars as a function of period. The 20 brightest RR Lyrae stars have visual magnitudes in the range from 7 to 10 . Parallax measurements are needed at the $1 \%$ level which corresponds to 40 microarcseconds for RR Lyrae itself. Distances to nearby subdwarfs in 
the magnitude range of 7.2 - 12 would calibrate the subdwarf luminosities which are used in fitting globular cluster main sequences. Parallaxes are needed with 30 microarcsecond precision. Parallaxes of bright, visual magnitude $10, \mathrm{~K}$ giants in the galactic disk, accurate at 50 microarcseconds, would probe the dark matter in our galaxy.

With 100 microarcsecond measurements and a sufficient mission duration, Newcomb could detect Jupiter-sized planets around nearby stars. The problem would be whether the 30-month lifetime envisioned for Newcomb would be long enough to detect the quadratic term in the motion of a star necessary to indicate the existence of a planet.

Gravity Probe B, an experiment which measures the general relativistic frame dragging due to the spinning Earth, needs a bright guide star, Rigel, with the proper motion known in an inertial frame to approximately 1 milliarcsecond per year or better. Newcomb could determine the proper motion of Rigel relative to a few bright quasars tied directly to the reference frame.

Newcomb will provide, for a limited number of stars, parallaxes and proper motions which are an order of magnitude more accurate than Hipparcos.

\section{Satellite Characteristics}

The satellite orbit would be approximately 600 nautical miles altitude, Sun synchronous, with continuous Sun illumination and $90^{\circ}$ inclination. The payload weight would be in the range of 150 to 200 pounds, with a total satellite weight in the $500-600$ pounds range. It could be launched from a Pegasus launch vehicle, or share an Argos mission. Approximate size would be 3.2 cubic feet. Electric power would be 28 volt DC with approximately 125 Watts average power. Attitude control based on the step bus would be $\pm 0.5^{\circ}$ in inertial orientation. The object payload with gimbals would provide $\pm 0.001^{\circ}$ to $\pm 0.0001^{\circ}$. Thermal control would be a passive system for the spacecraft, with inertial attitude and sun soaker characteristics, and active thermal control for the critical optical elements.

\section{Sensor Description}

The Newcomb Astrometric Satellite would have a stacked set of 3, or 4, Michelson optical interferometers oriented at $0^{\circ}, 40.9^{\circ}$, and $60.5^{\circ}$. Selected star pairs would be measured one by one. Free axis pointing of the optics payload would be used to acquire each star pair. The dispersed fringe pattern from the interferometer would be detected by a two-dimensional detector made up of $8000 \times 32$ elements, so the detector would work in a wide frequency band from 4000 to 8000 angstroms. The size of the detec- 
tor and the small optics provide a field of view of several arcminutes. The detectors would do onboard image integration for the fringe detection. The stellar separations and instrument parameters would be determined from the data reduction process with the long-term 10 - 20 hour drifts in instruments calibrated by the observations. The interferometer would use a $30 \mathrm{~cm}$ baseline with an aperture of $2 \times 5 \mathrm{~cm}$. The instrument field-of-view would be approximately $0.7^{\circ}$ with a sensitivity of approximately 15 th magnitude.

\section{Technical Issues}

The preliminary mission concept has been completed primarily based on the POINTS concept. The critical technical issues which have been identified are as follows:

Thermal control is necessary in order to maintain the stability of the optics payload.

The detectors necessary for the fringe detection must be identified.

The attitude control and payload pointing requirements must be achieved. Gimbals can provide $0.001^{\circ}$ of arc while the payload pointing requires $0.0001^{\circ}$.

The optimum on-board fringe detection and data processing must be developed.

Mission planning must be accomplished to develop the observing mode and the catalog of target stars.

Finally, a detailed optical design trade-off study must be done to specify the optics sizes necessary to achieve a signal-to-noise ratio required for a successful mission.

\section{Summary}

In summary, the Newcomb Astrometric Satellite is a concept for a small, quick, inexpensive, initial optical interferometer in space. It could measure the angles between stars, to define a inertial reference frame with the precision of 0.1 milliarcsecond. By means of this observational capability, proper motions and parallaxes can be determined and other scientific investigations achieved.

\section{References}

Reasenberg, Robert D., Babcock, Robert W., Phillips, James D., Johnston, Kenneth J., and Simon, Richard S. (1993) "Newcomb, A POINTS Precursor Mission with Scientific Capacity" in Spaceborne Interferometry, Proceedings of SPIE Conference, 1947.

Fey, A., Russell, J. L., Ma, C., Johnston, K. J., Archinal, B.A., Carter, M.S., Holdenreid, E., and Yao, Z. (1992) Astron J. 104, 891. 